\title{
Investigating the association of atopy and aeroallergen sensitization and chronic spontaneous urticaria
}

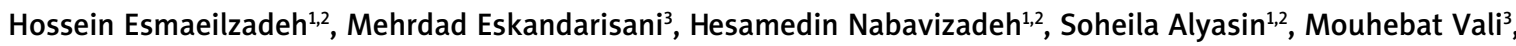 \\ Negar Mortazavi ${ }^{4}$
}

${ }^{1}$ Allergy Research Centre, Shiraz University of Medical Sciences, Shiraz, Iran

2Department of Allergy and Clinical Immunology, Namazi Hospital, Shiraz University of Medical Sciences, Shiraz, Iran

${ }^{3}$ Student Research Committee, Shiraz University of Medical Sciences, Shiraz, Iran

${ }^{4}$ Department of Clinical Pharmacy, School of Pharmacy, Shiraz University of Medical Sciences, Shiraz, Iran

Adv Dermatol Allergol 2022; XXXIX (1): 121-125

DOI: https://doi.org/10.5114/ada.2022.113805

\begin{abstract}
Introduction: Chronic spontaneous urticaria (CSU) is a common skin allergic reaction with an unknown mechanism. There is inadequate evidence to support the role of aeroallergen sensitization in CSU.

Aim: The present study was conducted to investigate the association between the aeroallergen sensitivity and CSU. Material and methods: In this cross-sectional study, 91 patients with CSU were recruited. Information on demographic characteristics was collected. The skin prick test (SPT) for most prevalent aeroallergens and pulmonary function test (PFT) were performed and the results were clinically matched to investigate the allergic rhinitis (AR) and asthma. Total serum immunoglobulin E (IgE) concentration was analysed in the subjects for the presence of atopy. Results: Ninety one patients (75 of them were females) were enrolled in the study (with a mean age of $36.90 \pm 12.93$ years). SPT result was positive in 75 ( $82.4 \%$ ) subjects. Weeds (67 cases, $73.6 \%$ ), trees (61 cases, $67 \%$ ), and dust mites ( 55 cases, $60.4 \%$ ) were the most common pollens in the positive SPT samples. Thirty-seven (40.7\%) of the patients had allergic rhinitis. There was a statistically significant association between the presence of AR and positive SPT result $(p=0.002)$. Eight patients had allergic asthma and $45(49.4 \%)$ of the patients had a total serum IgE level of more than $100 \mathrm{IU} / \mathrm{ml}$.

Conclusions: Our results indicated a clear association between the presence of aeroallergen sensitivity and development of CSU. AR exists as a co-morbidity of CSU.
\end{abstract}

Key words: urticaria, atopy, aeroallergens, allergy, skin prick test.

\section{Introduction}

Urticaria or hives is a common skin reaction characterized by the three typical features including the central swelling (wheal) usually surrounded by the erythema, pruritus and a time course of 30 min to $24 \mathrm{~h}$ when the skin returns to the previous normal form without any sequela. Chronic urticaria (CU) is defined as the existence of recurrent urticaria, angioedema, or both of them on most days of the week, lasting for 6 weeks or longer affecting $0.5-1 \%$ of the population [1]. CU is more common in the adults than children with a female to male ratio of $2: 1$ and it ordinarily affects the people in the third to fifth decades of their life [1, 2]. About $20 \%$ of the patients with CU have physical reproducible triggers such as vibration, water, prolonged pressure, cold, heat, and exercise classifying as the physical urticaria. Remaining patients (80\%) are classified as the cases with chronic idiopathic urticaria (CIU) that except the subgroup of chronic autoimmune urticaria have no definite identified allergic cause or contributing factor and they are known as the patients with chronic spontaneous urticaria (CSU) [2, 3]. Although there are several theories about the pathogenesis and aetiology of CSU, there is no established theory yet, and aetiology of CSU cannot be identified in most cases [2-4]. Among different possible causes for development of CSU (e.g., autoimmunity, serum plasma factors, defects in the skin mast cells and/or basophils, infectious agents, foods or food additives, and medication), atopy and sensitivity to inhalant allergens (aeroallergens) triggering the allergic reactions such as allergic rhinitis (AR) or asthma

Address for correspondence: Hossein Esmaeilzadeh MD, Allergy Research Centre, Shiraz University of Medical Sciences, Mohammad Rasool Allah Research Tower, Khalili Street, Shiraz, Iran, P.O. Box: 719335899, fax: +98 71-36474298, e-mail: esmailzadeh_ho@yahoo.com; Negar Mortazavi (Pharm D), Department of Clinical Pharmacy, School of Pharmacy, Shiraz, Iran, e-mail: mortazavi_ne@yahoo.com Received: 7.06.2020, accepted: 14.11.2020. 
have attracted a great deal of attention [5-8]. Patients experiencing food or drug lgE-mediated reactions frequently demonstrate the urticarial reactions; on the other hand, frequency of the hives in the patients with CSU fluctuates over days and seasons. This highlights the need for studying the possible association and role of atopy and aeroallergens in development of CSU. Caliskaner et al., in a study, found a higher ratio of skin prick test (SPT) reactivity to house dust mites in the patients with CU than the healthy controls but this ratio was not statistically significant [7]. Refaat et al. concluded that the sensitivity to mites and aeroallergens should be considered as a probable cause for formation of CSU [8]. However, there are limited studies in this context and there is inadequate evidence to support the role of atopy in development of CSU.

\section{Aim}

Therefore, the present study was conducted to investigate the association and existence between the presence of atopy and aeroallergen sensitivity and presentation of CSU.

\section{Material and methods}

Ninety-one patients with CSA were recruited in this study from 138 patients with CU referred to the Imam Reza Outpatient Clinic, Shiraz, Iran from September 2018 to January 2020. A specific questionnaire was completed and recorded for the patients to collect the information on the demographic characteristics, medication history, allergic disease history (asthma, AR, atopic dermatitis, anaphylaxis, angioedema, etc.), triggers of hives, and duration of the disease. Diagnosis of AR was made if there were both positive SPT result and history of rhinitis symptoms in exposure to different pollens. Patients with a vague history were followed up over different seasons and times to investigate the symptoms after exposure. Asthma diagnosis was confirmed by the pulmonary function test (PFT).

\section{Inclusion criteria}

Patients (aged $\geq 12$ years old) who met the CU diagnostic criteria according to the allergy and immunology specialists were recruited in this study, those with the history of CU and/or angioedema presenting the symptoms on most days of the week, over at least 6 weeks were also included. Informed written consent was obtained from each subject.

\section{Exclusion criteria}

Patients with one or more of the following criteria were excluded from the study: chronic inducible physical urticaria, hereditary/acquired angioedema, urticarial vasculitis, pregnancy, anaphylaxis, drug reactions, IgEmediated food or drug allergy, chronic or systemic diseases (such as cardiovascular, respiratory, renal, hepatic, haematological, thyroid, and other skin diseases or malignancy, etc.), and smokers.

Consumption of the nonsteroidal anti-inflammatory drugs (NSAIDs) and alcohol, as CU triggers, was prohibited throughout the study.

\section{Interventions}

SPT was performed using the outdoor and indoor aeroallergen extracts (Greer Company, USA). According to the most prevalent allergens in the country, tree mix, birch, grass mix, weed mix, Russian thistle, alternaria, mould mix, pets (cat and dog), and dust mites were selected for the SPT. A wheal of at least $3 \mathrm{~mm}$ diameter and/or a flare of at least $10 \mathrm{~mm}$ diameter were considered as a positive result.

The total serum lgE level was measured using the commercial enzyme immunoassay and lgE of more than 100 IU/ $\mathrm{ml}$ was considered as positive.

PFT was performed to investigate the asthma diagnosis. A $15 \%$ or more decrease in the forced expiratory volume at the end of first second $\left(\mathrm{FEV}_{1}\right)$ after exercise or $12 \%$ and more increase in it after salbutamol administration was diagnosed as asthma.

\section{Ethics}

The Ethics Committee of the Shiraz University of Medical Sciences approved the protocol of the study by number 1398.449.

\section{Statistical analysis}

Data were analysed using the SPSS software (SPSS, version 19) and GraphPad software was used to draw the charts. Data were expressed as frequency (percentage). Qualitative variables were compared using the $\chi^{2}$ test. A $p$-value of less than 0.05 was considered as statistically significant.

\section{Results}

In this study, 91 patients were recruited based on the aforementioned criteria. The mean age of them was $36.90 \pm 12.93$ years. Most of the subjects were female (75 cases, 82.4\%). Allergic diseases including AR, asthma, eczema, and angioedema were more prevalent in the females (Figure 1) however, the difference was not statistically significant ( $p \geq 0.05)$. AR (48\%) and angioedema (22.7\%) were the most prevalent co-morbid allergic diseases.

SPT was positive for the aeroallergens in 75 (82.4\%) of the patients. However, 24 (26.4\%) of them were positive in their skin test for each of the six allergens mentioned in the study (Figure 2), and in general, the skin test was positive for more than two allergens in 72 (79.1\%) of the patients. The highest prevalence of sensitization to the aeroallergens in SPT was shown for the weeds (67 cases, $73.6 \%$ ), trees (61 cases, $67 \%$ ), dust mites ( 55 cases, $60.4 \%$ ), moulds (50 cases, $54.9 \%$ ), pets (47 cases, $51.6 \%$ ), 
A
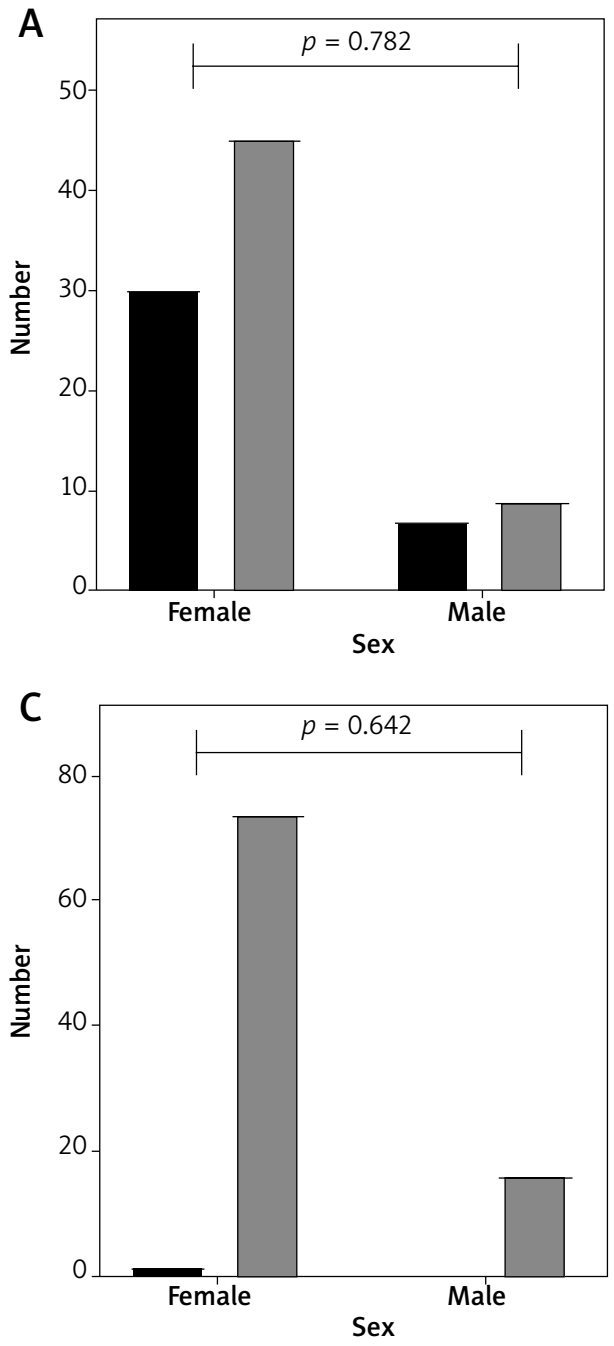
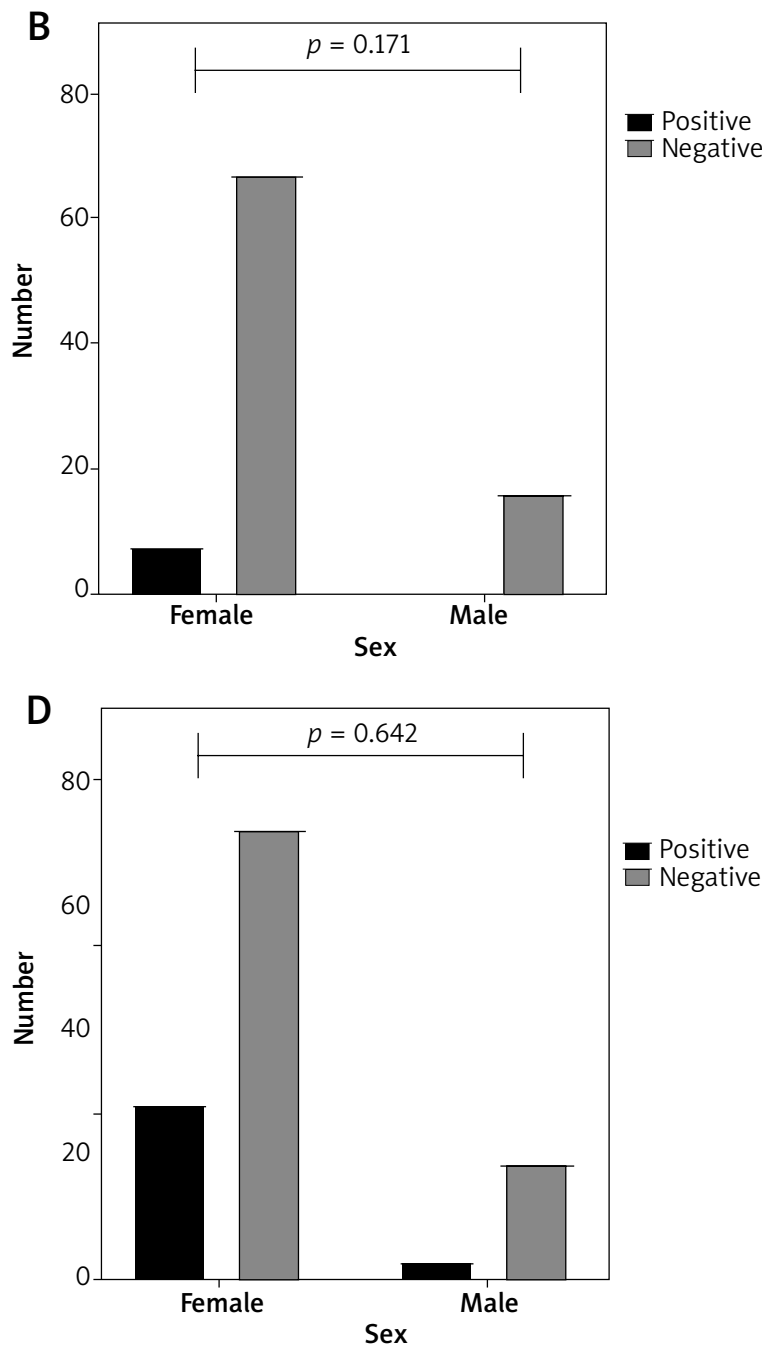

Figure 1. Detection of allergic rhinitis (A), asthma (B), eczema (C) and angioedema (D) in 91 patients with chronic spontaneous urticaria by sex

and grass (41 cases, 45.1\%). All the patients with asthma had positive SPT as well as AR.

Among 75 patients with positive SPT, only 36 (48.0\%) of them had AR symptoms after exposure to seasonal or indoor aeroallergens and met the diagnostic criteria of AR. On the other hand, AR was positive in 37 (40.7\%) of the patients meaning that 1 patient had a history of AR with a negative SPT result. There was a statistically significant relationship between the presence of $A R$ and positive SPT result ( $p \leq 0.05)$ (Table 1$)$. Forty-five (49.4\%) patients had a total serum IgE level of more than 100 $\mathrm{IU} / \mathrm{ml}$ among which 6 patients had negative SPT. Table 1 shows the association between the various kinds of allergic disease and SPT results.

All the patients received Fexofenadine as a $\mathrm{H} 1$-antihistamine and Doxepin was used in 21 (23.1\%) and 61 $(67.0 \%)$ of the patients. Figure 3 shows the information of other administered drugs.

\section{Discussion}

CSU aetiology is still unknown. Recent researches have mostly focused on the underlying immune mechanisms, like mediators and mast cell activation pathways [4]. Symptoms and severity of CSU fluctuate over days and times; on the other hand, IgE-mediated aeroallergen sensitization results in the mast cell activation after exposure. Thus, the current study was performed to investigate the association between the aeroallergen sensitization and development of CSU.

In our study, SPT was positive in more than $80 \%$ of the patients indicating the sensitization to the aeroallergens, while only $40 \%$ of the patients with CSU had proven AR. The difference is due to the different nature of sensitization and allergy. The association between the positive SPT result and induction of the symptoms after exposure is necessary to confirm the diagnosis of AR [9]. 


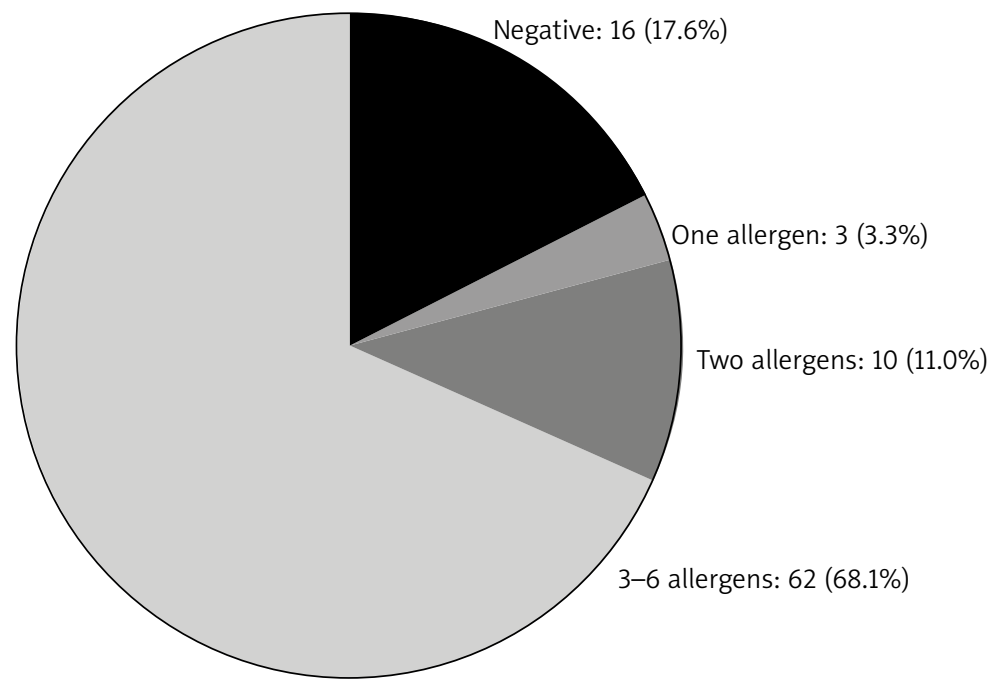

Figure 2. Distribution of aeroallergen sensitization

Our findings revealed that weeds, trees and mites were the most prevalent aeroallergens in the positive SPT result while only $48 \%$ of the patients with positive SPT had clinically relevant AR. The pattern of sensitization to the aeroallergens in our study is compatible with previous findings reporting the weeds as the most prevalent aeroallergen in our country as a cause of AR [10, 11]. A high rate (80\%) of the SPT sensitization observed in our study among the patients with CSU has not been previously reported in the literature on normal population or the patients with other allergic diseases, which can be due to the unknown mechanism of CSU.

$A R$ and CSU presentations result from the mast cell activation and release of the mediators and cytokines; although it seems that AR is IgE-mediated but CSU is not at all [12]. There is limited evidence on the role of aeroallergens in development of CSU. Caliskaner et al. found

Table 1. Detection of allergic rhinitis, asthma, eczema and angioedema in ninety one patients with chronic spontaneous urticaria

\begin{tabular}{|c|c|c|c|c|}
\hline \multirow[t]{2}{*}{ Variable } & & \multicolumn{2}{|c|}{ SPT } & \multirow[t]{2}{*}{$P$-value ${ }^{\star}$} \\
\hline & & Positive & Negative & \\
\hline \multirow{2}{*}{$\begin{array}{l}\text { Allergic } \\
\text { rhinitis }\end{array}$} & Positive & $36(48.0 \%)$ & $1(6.3 \%)$ & \multirow[t]{2}{*}{0.002} \\
\hline & Negative & $39(52.0 \%)$ & 15 (93.8\%) & \\
\hline \multirow[t]{2}{*}{ Asthma } & Positive & 8 (10.7\%) & $0(0.0 \%)$ & \multirow[t]{2}{*}{0.171} \\
\hline & Negative & 67 (89.3\%) & $16(100.0 \%)$ & \\
\hline \multirow{2}{*}{$\begin{array}{l}\text { Atopic } \\
\text { dermatitis }\end{array}$} & Positive & $1(1.3 \%)$ & $0(0.0 \%)$ & \multirow[t]{2}{*}{0.642} \\
\hline & Negative & 74 (98.7\%) & 16 (100.0\%) & \\
\hline \multirow{2}{*}{$\begin{array}{l}\text { Urticarial } \\
\text { angioedema }\end{array}$} & Positive & 17 (22.7\%) & $6(37.5 \%)$ & \multirow[t]{2}{*}{0.215} \\
\hline & Negative & 58 (77.3\%) & $10(62.5 \%)$ & \\
\hline
\end{tabular}

${ }^{*} \chi^{2}$ test. a higher ratio of SPT reactivity to house dust mites in patients with CU than the healthy controls but this ratio was not statistically significant enough to show the strong relation with CSU [7]. Refaat et al., in a study on 200 patients with CSU, concluded that sensitization of the mites and aeroallergens should be considered as a probable cause of development of CSU and they found no associated allergic respiratory diseases after performing SPT [8]. The results of our study showed the mites as the third most common allergen in CSU. This contradiction is due to the difference in the regional and climate situation as most parts of our country have dry and warm weather that is not ideal for dust mites.

All the 8 patients with asthma in our study had positive SPT and all of them had AR meaning that they had allergic asthma, which is in line with other studies showing that seasonal exacerbation of allergic asthma can

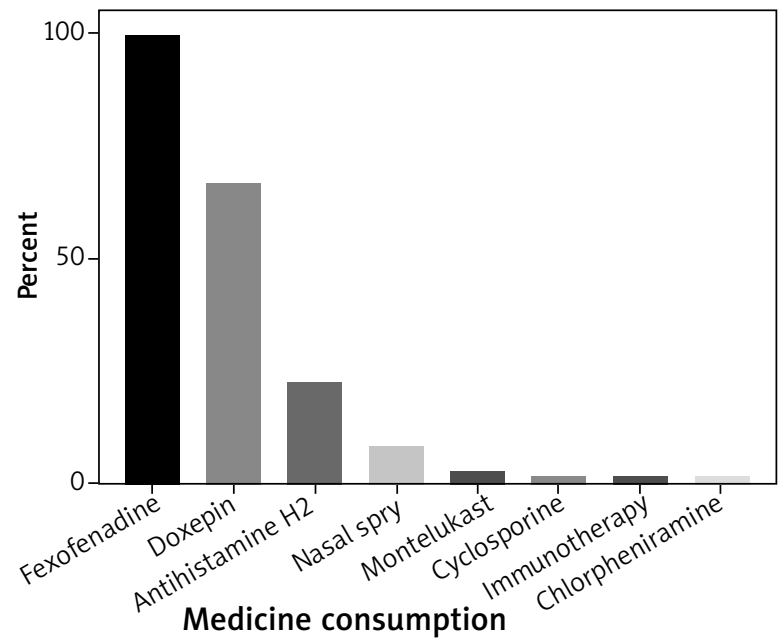

Figure 3. Percent of medicine concumption in participant 
trigger recurrent episodes of acute urticaria [13], but joint autoimmune mechanism is the most likely cause of asthma in CSU [14]. Almost all the patients with atopic dermatitis (AD) had negative SPT at the same time, which is compatible with non-IgE mediated pathophysiology of $A D$ and again autoimmune mechanism can be the cause of this co-morbidity [14]. There was no statistically significant relationship between the presence of asthma and $A D$ and the development of CSU.

Raciborski et al. reported the prevalence of angioedema of $3 \%$ of the patients with acute and chronic urticaria [15], but our findings revealed its prevalence of $22.7 \%$, which is higher than the mentioned study. This high rate may be caused by genetic or ethnicity-related factors. Gao et al. indicated that a high serum IgE level is associated with the disease severity as well as with low sensitivity to antihistamine treatment of the patients [16]. The prevalence of angioedema as a presentation of CSU was reported by $22.7 \%$ in our study. This higher rate compared to previous studies can be attributed to the study population as in our study, just the patients with CSU were included and those with other forms of CU and different ethnicity were not included.

Administering a high dose of second-generation $\mathrm{H} 1$ antihistamines is considered as the first-line treatment in CU. $\mathrm{H} 2$ antihistamines, cyclosporine and montelukast are among some drugs used in the patients with antihistamine-resistant CU. The medication record obtained in our study is in line with treatment guidelines of CU [17].

There were some limitations in this study. Firstly, our results were not based on a comparison between the healthy control group and the patient group, thus our results were compared with the literature and data in normal population to resolve this limitation. Secondly, this study was carried out in one centre located in southwest of the country to investigate the effect of pollens and aeroallergens. Therefore, multicentre studies with a larger sample size are recommended with an exact pollination schedule. Our study shows the association of atopy and aeroallergen sensitization with CSU and no cause-and-effect relationship.

\section{Conclusions}

Our results indicated a clear association between the presence of aeroallergen sensitivity and CSU. A high rate of AR prevalence in our study introduces it as a co-morbid condition in CSU. Allergy and IgE-mediated reactions can exist in CSU.

\section{Acknowledgments}

This work was supported by the Shiraz University of Medical Sciences (Grant number 97-01-01-19071).

\section{Conflict of interest}

The authors declare no conflict of interest.

\section{References}

1. Vestergaard C, Deleuran M. Chronic spontaneous urticaria: latest developments in aetiology, diagnosis and therapy. Ther Adv Chronic Dis 2015; 6: 304-13.

2. Ferrer M. Epidemiology, healthcare, resources, use and clinical features of different types of urticaria. Alergológica 2005. J Investig Allergol Clin Immunol 2009; 19 (Suppl 2): 21-6.

3. Zuberbier T, Aberer W, Asero R, et al. The EAACI/GA²LN/ EDF/WAO guideline for the definition, classification, diagnosis and management of urticaria. Allergy 2018; 73: 1393-414.

4. Kaplan A, Greaves M. Pathogenesis of chronic urticaria. Clin Exp Allergy 2009; 39: 777-87.

5. Gaig P, Olona M, Lejarazu DM, et al. Epiodemiology of urticaria in Spain. J Investig Allergol Clin Immunol 2004; 14: 214-20.

6. Lapi F, Cassano N, Pegoraro V, et al. Epidemiology of chronic spontaneous urticaria: results from a nationwide, population-based study in Italy. Br I Dermatol 2016; 174: 996-1004.

7. Caliskaner Z, Ozturk S, Turan M, Karaayvaz M. Skin test positivity to aeroallergens in the patients with chronic urticaria without allergic respiratory disease. J Investig Allergol Clin Immunol 2004; 14: 50-5.

8. Refaat M, Ossman E, Farres M, et al. Assessment of the role of aeroallergens in patients with chronic urticaria. Rev Franç d'Allergol 2010; 50: 394-7.

9. Migueres M, Dávila I, Frati F, et al. Types of sensitization to aeroallergens: definitions, prevalences and impact on the diagnosis and treatment of allergic respiratory disease. Clin Transl Allergy 2014; 4: 16.

10. Oskouei YM, Hosseini RF, Ahanchian H, et al. Report of common aeroallergens among allergic patients in northeastern Iran. Iran J Otorhinolaryngol 2017; 29: 89.

11. Moghtaderi M, Teshnizi SH, Farjadian S. Sensitization to common allergens among patients with allergies in major Iranian cities: a systematic review and meta-analysis. Epidemiol Health 2017; 39: e2017007.

12. Vonakis BM, Saini SS. Basophils and mast cells in chronic idiopathic urticaria. Curr Allergy Asthma Rep 2005; 5: 270-6.

13. Vadasz Z, Kessel A, Hershko AY, et al. Seasonal exacerbation of asthma is frequently associated with recurrent episodes of acute urticaria. Int Arch Allergy Immunol 2016; 169: 263-6.

14. Tedeschi A, Cottini M, Asero R. Simultaneous occurrence of chronic autoimmune urticaria and non-allergic asthma: a common mechanism? Eur Ann Allergy Clin Immunol 2009; 41: 56-9.

15. Raciborski F, Kłak A, Czarnecka-Operacz M, et al. Epidemiology of urticaria in Poland - nationally representative survey results. Adv Dermatol Allergol 2018; 35: 67-73.

16. Gao YX, Han Y, Yao X. Elevated serum total IgE levels in patients with chronic urticaria indicate insensitivity to antihistamine treatment. Int J Dermatol Venereol 2019; 2: 145-9.

17. Zuberbier T, Asero R, Bindslev-Jensen C, et al. EAACI/ GA2LEN/EDF/WAO guideline: definition, classification and diagnosis of urticaria. Allergy 2009; 64: 1417-26. 